\title{
Experimental Study of Hydrodynamics in the Aquarium Using PIV Method
}

\author{
Djimako Bongo ${ }^{1,}$, Alexis Mouangué Nanimina ${ }^{1}$, Edith Kadjangaba ${ }^{2}$, Jean-Yves Champagne ${ }^{3}$ \\ ${ }^{1}$ Department of Mechanical Engineering, Higher National Institute of Science and Technology of Abéché, Abéché, Ouaddaï, Chad \\ ${ }^{2}$ Department of Hydrogeology, Faculty of Exact and Applied Sciences, University of N'Djamena, N'Djamena, Chad \\ ${ }^{3}$ Laboratory of Fluid Mechanics and Acoustics, INSA-Lyon, Lyon, France
}

\section{Email address:}

djimako.b5@gmail.com (D. Bongo), alexisnanichad@gmail.com (A. M. Nanimina), amnanimina@yahoo.fr (A. M. Nanimina), edith.kadjangaba@gmail.com (E. Kadjangaba)

${ }^{*}$ Corresponding author

\section{To cite this article:}

Djimako Bongo, Alexis Mouangué Nanimina, Edith Kadjangaba, Jean-Yves Champagne. Experimental Study of Hydrodynamics in the Aquarium Using PIV Method. American Journal of Energy Engineering. Vol. 7, No. 4, 2019, pp. 74-79. doi: 10.11648/j.ajee.20190704.11

Received: October 12, 2019; Accepted: November 20, 2019; Published: December 4, 2019

\begin{abstract}
The purpose of this study is to determine the phase indicator functions (vacuum rate, velocity and bubble size) of the gas-liquid flow. The gas-liquid flows in these columns (aquarium) are intrinsically unstable and the dynamics of such flows influence the mixing and mass transfer performance. It is therefore important to characterize the dynamics of gas-liquid flow. Also, the complete knowledge of the global dynamics of the fluids of the bubble column is based on that of the bubble. The experimental analysis is carried out using a two-phase instrumentation consisting of an optical fiber bi-probe. The use of the experimental techniques has enabled a better understanding of the hydrodynamics of two-phase flow. In terms of results, intrusive techniques provide local measurements while non-intrusive techniques provide a distribution over a cross-section with different spatial and temporal resolutions. The optical fiber bi-probe placed between two column flanges permit to have a complete mapping of the dispersed phase flow. The use of a mass flow meter and an ultrasonic flow meter, in different flow configurations, made it possible to obtain data on the operation of the column. However, the analysis of granulometry of the bubbles in the columns is performed by intrusive, flow-disrupting and non-intrusive techniques. Knowledge of bubble size and vacuum rate is crucial for determining interfacial air.
\end{abstract}

Keywords: Flow, Hydrodynamics, PIV, Aquarium, Bubbles

\section{Introduction}

Flow visualization provides insight into the interpretation of experimental results with respect to local measurements (PIV). The advantages and disadvantages of the various intrusive and non-intrusive techniques and their applications to multiphasic flows have been presented by several previous works [1-3]. Indeed, as we will detail in this study, Taylor's flow is sensitive to its history, which raises the question of the reproducibility of the experiments and the measurements obtained. Also, to answer this problem, a technique of visualization of the flow was used in our experiment to determine experimentally the fields of velocity, the diameters of the bubbles of $\mathrm{CO}_{2}$ and the rate of vacuum following the vertical one in an aquarium allowing to select the desired hydrodynamic regime [4-6].

\section{Description of the Experience}

\section{Principle}

The technique consists in seeding the flow with DANTEC DYNAMICS particles. These particles are spherical crystals HGS-10, hollow $\varnothing 10 \mu \mathrm{m}$. They are used at a wavelength around $500 \mu \mathrm{m}$. It is a crystalline microscopic powder with a diameter of $50 \mu \mathrm{m}$ which has the particularity of aligning itself along the current lines. In the presence of an incident light, they reflect the light with deferential intensities according to the exposed surface. This gives an idea of the general topology of the flow.

A camera placed perpendicularly to the measurement plane 
records the snapshots of the plotters. The images are then divided into interrogation cells. A cell of an image is mapped to the same cell in the twin image. An intercorrelation is then applied between the two cells, and the position of its maximum gives the value of the most probable displacement of all the particles. This procedure, applied to all the interrogation cells, makes it possible to obtain the projection of a velocity vector field. The acquisition of numerous images will make it possible to calculate statistical magnitudes of the 1000 images for the speed in the studied plane. Which did not make it possible to do the image processing and also with the calculations of the averages and to remove it from the scrambles.

\section{Materials and Protocol}

\subsection{Materials}

The following materials are used to carry out the experiments at the fluid mechanics laboratory of INSA Lyon.

Aquarium size $25 \times 25 \times 70 \mathrm{~cm}$;

Laser Neodymium-doped Yttrium Aluminum Garnet;
99.7\% pure $\mathrm{CO}_{2}$ bottle;

$\mathrm{PC}$ for recording purpose;

FastCam Photron SA4 camera;

NAVITAR Zoom 6000 lens;

Syringe Pump Lambda Vit-Fit;

High precision scale balance (up to $200 \mathrm{~g}$ ).

\subsection{Protocol}

1. Filling $15 \mathrm{~L}$ of fresh water in the aquarium;

2. Positioning the camera facing the aquarium and connect it to the computer;

3. Lights illuminated on the aquarium;

4. Priming the $\mathrm{CO}_{2}$ flow by the syringe pump, at a speed of $0.8 \mathrm{~mm} / \mathrm{min}$;

5. Adjusting the focal length of the lens relative to the laser plane;

6. Camera settings (acquisition rate and resolution);

7. Visualization of the bubbles on the screen, via the Photron software of the camera.

Figure 1 presents the experiment apparatus used for the experiment.

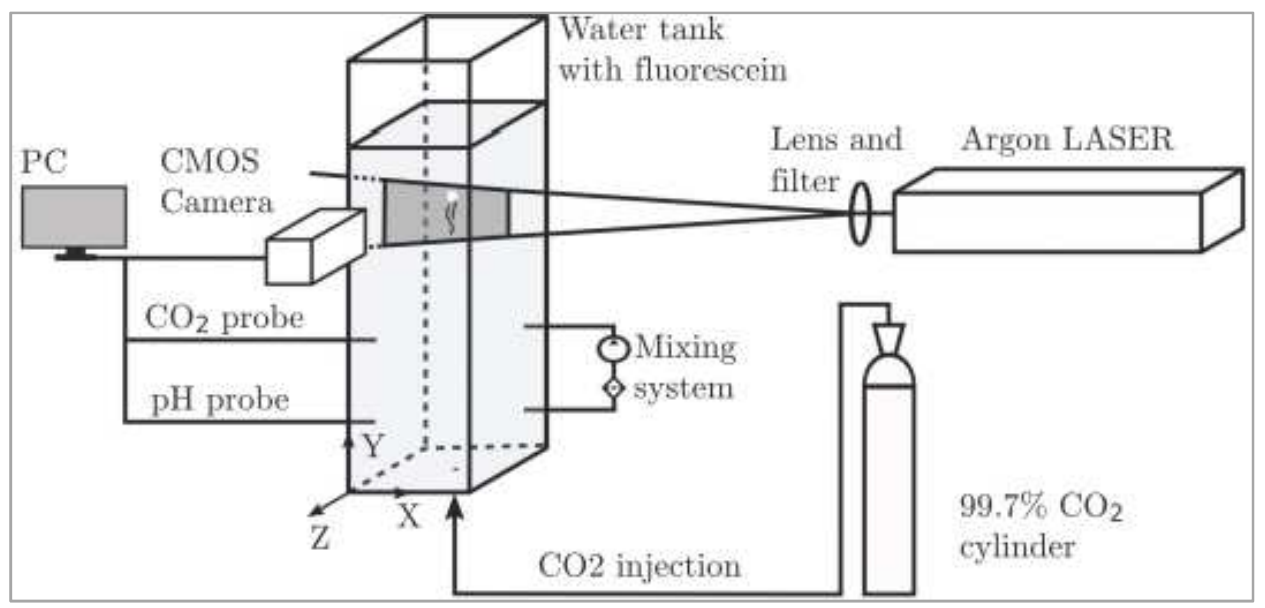

Figure 1. Experimental apparatus.

\subsection{Fluorescein-Water Mixture}

In the tank (figure 1) is poured a precise amount of water (demineralized water to eliminate any unknowns that could interfere in the study of the dissolution of $\mathrm{CO}_{2}$ ), quantity measured using a volumetric flask of $2000 \mathrm{ml}$ allowing to control at then the concentration of fluorescein to be poured. The fluorophore is then added to the water using a graduated pipette.

Once the substances are added, a B. Kern pump connected to the tank will suck and spit the mixture at two different places, allowing the liquid to be mixed cleanly so that it is homogeneous.

Light can be absorbed by chromophores and / or fluorophores. Fluorophore is a chromophore that emits photons when it absorbs light (figure 2). The absorption of a photon allows the passage of the chromophore from the fundamental energetic state to an excited state [7].

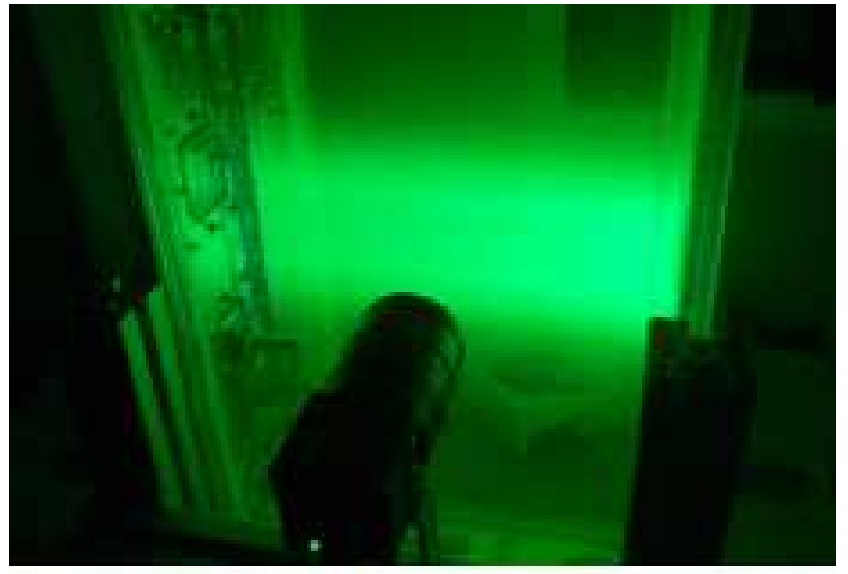

Figure 2. Visualization of the laser plane by the camera.

Beer-Lambert Law

When a beam of light passes through a solution of an 
absorbing substance, it undergoes an absorption which is described by the Beer-Lambert law (Equation 1):

$$
\frac{I_{(x)}}{I_{(x=0)}}=\operatorname{expo}\left(2,303 \int_{0}^{x} \epsilon(\lambda) C\left(x^{\prime}\right) d x^{\prime}\right)
$$

Where $I$ is the intensity of the light beam, $x$ the distance along the light propagation path or optical path $(x=0$ is an arbitrary choice), $\mathrm{C}$ the local concentration of the absorbing substance and $Q$ its coefficient of absorption. The BeerLambert law is valid for a parallel and monochromatic beam of light of wavelength $\lambda$. The molecular absorption coefficient $Q$ of the absorbing substance is a molecular property which reflects its ability to absorb a photon at a given wavelength.

\section{Results}

After each recording, a pattern figure 3 is placed in the aquarium instead of the diffuser, and an image is recorded in order to later measure the bubbles observed. The resolution is 1024 x 1024 for all shots. The chosen acquisition frequencies vary between $1000 \mathrm{img} / \mathrm{s}$ and $3000 \mathrm{img} / \mathrm{s}$ for the observation of moving bubbles, and is $50 \mathrm{img} / \mathrm{s}$ for the test pattern. The figure 3 shows the example of moving bubbles pattern.

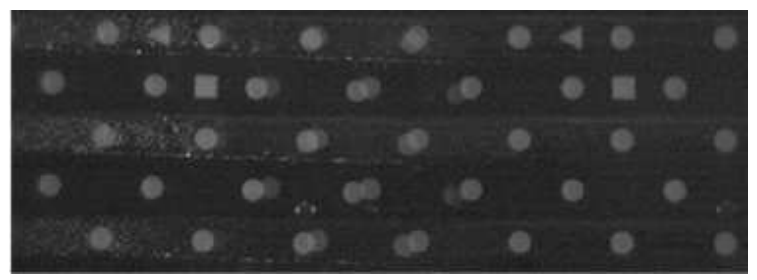

Figure 3. Example of the pattern.

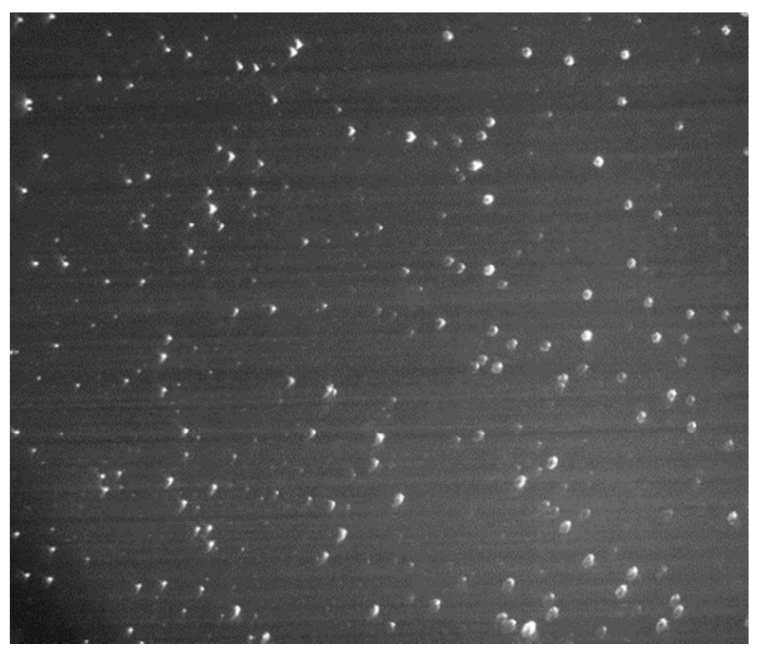

Figure 4. Averaged image with subtraction of porridge.

\section{Acquisition of images}

In order to obtain the images of the particles, the lighting of the flow is done using a laser sheet. This is produced by a lens system placed at the output of a Neodymium-doped Yttrium Aluminum Garnet laser source, emitting at a wavelength of $\lambda=532 \mathrm{~nm}$. The pulse energy of the laser used for PIV measurements is of the order of $25 \mathrm{~mJ}$. The interest of the laser pulses is to avoid the drag effect related to the displacement of the particles during the exposure time of the camera. The images obtained enable to very clearly observe the crystals figure 4 . The fast camera and its objective are much more powerful and high-performance to observe such small crystals.

\subsection{Vacuum Rate}

The analysis of figure 5 shows that the void fraction is directly proportional to the flow rate of $\mathrm{CO}_{2}$ injected into the aquarium. For each $\mathrm{CO}_{2}$ flow, the void ratio is relatively radially.

Gas retention is evaluated by static pressure measurements at different levels in the aquarium. These pressures are evaluated by a differential pressure probe $( \pm 1 \mathrm{mbar}$ ) (Figure 1 ). The gas retention is then calculated by the method of [8] given by the equation. (2):

$$
\varepsilon_{G}=1-\frac{\Delta P}{\rho_{L} g h}
$$

considering a density of the equal mixture:

$$
\rho_{m}=\rho_{G} \varepsilon_{G}+\rho_{L} \varepsilon_{L}=\rho_{L}\left(1-\varepsilon_{G}\right)
$$

where $\varepsilon \mathrm{G}$ is the gas retention in the aquarium, $\varepsilon \mathrm{L}$ is the liquid retention in the aquarium, $\Delta \mathrm{P}$ is the pressure decrease between two measurements $(\mathrm{Pa}), \mathrm{h}$ is the vertical height between two measurements $(\mathrm{m}), \rho \mathrm{L}$ is the density of the liquid phase $\left(\mathrm{kg} . \mathrm{m}^{-3}\right), \rho \mathrm{G}$ that of the gas phase $\left(\mathrm{kg} . \mathrm{m}^{-3}\right), \rho \mathrm{m}$ is the density of the mixture $\left(\mathrm{kg} . \mathrm{m}^{-3}\right)$ and $\mathrm{g}$ is the acceleration of gravity $\left(\mathrm{ms}^{-2}\right)$. At a given gas flow, the gas retention is a function inverse to the size of the bubbles and generally an increasing function of the air flow.

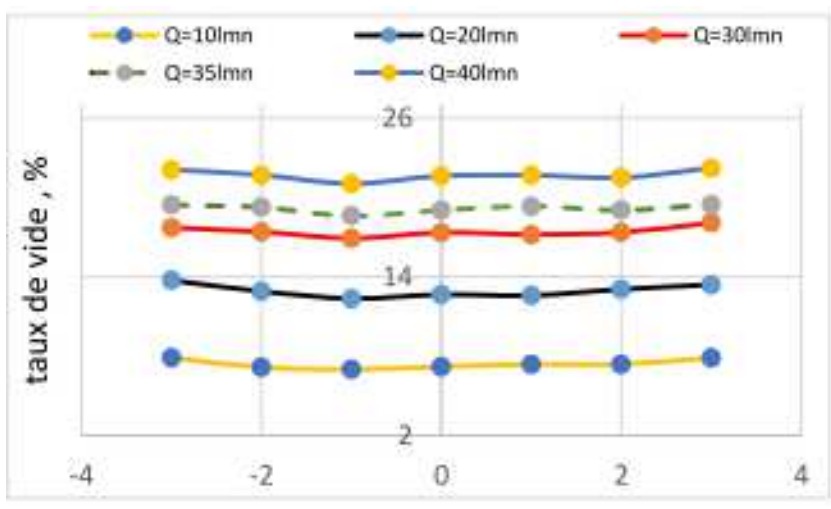

Figure 5. Evolution of the vacuum rate as a function of the injected $\mathrm{CO}_{2}$ flow rate.

\subsection{Bubble Size in Ascension}

The aquarium is equipped with measuring sockets at different heights to introduce an optical bi-probe whose principle and use have been described by [9]. This probe is capable of measuring average bubble diameters, their rate of rise and the rate of gas retention. To facilitate the 
interpretation of the data, certain hypotheses have been made: the movement of the bubbles is assumed exclusively vertical, the shape of the regular bubbles (spherical or ellipsoidal). However, with respect to the design of the probe, only bubbles larger than $2 \mathrm{~mm}$ could be taken into account in the evaluation of minimum diameter and average and maximum diameter (figure 6).

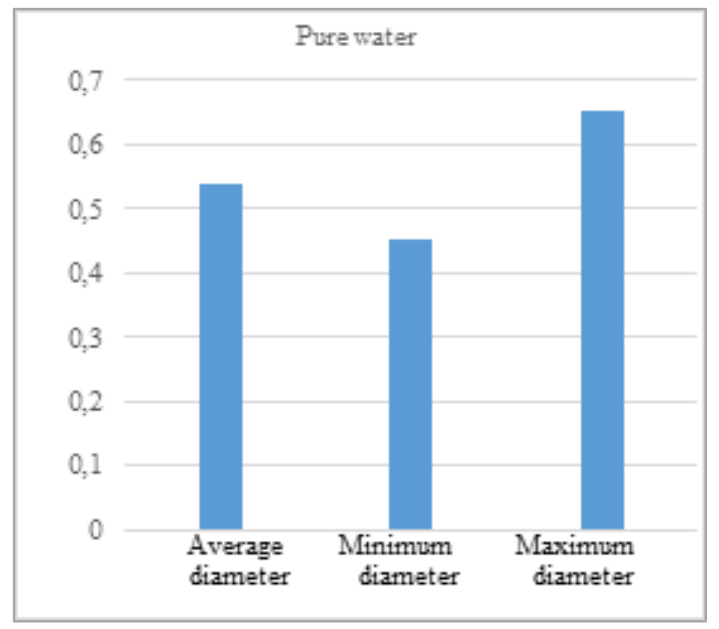

Figure 6. Size of the bubbles for the wooden diffuser.

\subsection{Effect of $\mathrm{CO}_{2}$ Flow on Bubble Size and Velocities}

The mean diameters of the bubbles provided by the different types of freshwater injector in the aquarium are shown in Figure 7. They increase with the flow of $\mathrm{CO}_{2}$ until reaching a limit value of about $0.7 \mathrm{~mm}$ [10]. In each case, the diameters of the bubbles are higher in fresh water. With a diffusion of the fine bubbles, the diameters measured by the probe are between 0.3 and $0.6 \mathrm{~mm}$ in fresh water, according to the air flow injected. With microbubble diffusion, the bubble diameters are between 0.4 and $0.6 \mathrm{~mm}$ in fresh water, and depending on the $\mathrm{CO}_{2}$ flow. The variation of this speed with respect to the injected air flow is presented in figure 8. In fresh water, the speed varies linearly with $\mathrm{HQ}$, between 0 and $0.7 \mathrm{~m} / \mathrm{s}$, whatever the type of injector used. The rise of microbubbles is slow compared to the fine bubbles in the aquarium.

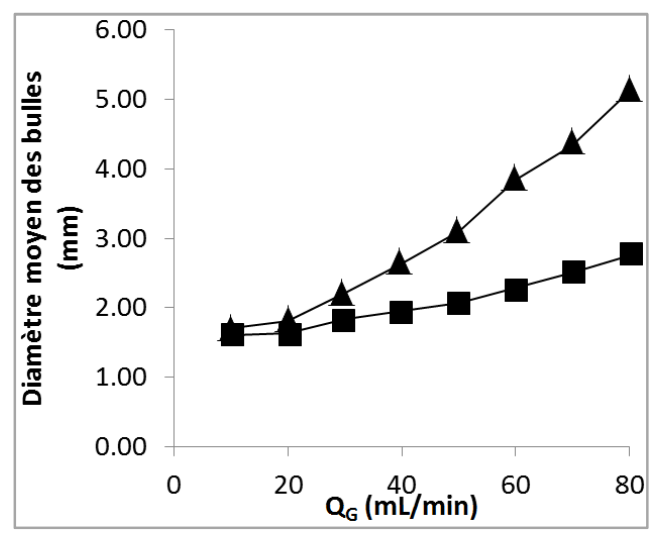

Figure 7. Average bubble diameter as a function of $\mathrm{CO}_{2}$ flow.

By neglecting the increase in speed due to the displacement of the water in the aquarium, the average speed of displacement of the bubbles can be calculated by the following relation:

$$
U_{G}=Q_{G} /\left(\varepsilon_{G} \cdot \Omega_{i}\right)
$$

where UG is the average bubble displacement velocity $\left(\mathrm{m} \cdot \mathrm{s}^{-1}\right)$, $\mathrm{QG}$ is the airflow $\left(\mathrm{m}^{3} \cdot \mathrm{s}^{-1}\right), \varepsilon \mathrm{G}$ is the gas retention and $\Omega \mathrm{i}$ is the cross section of the inner pipe $\left(\mathrm{m}^{2}\right)$.

The speed of movement of the bubbles is an increasing function of the air flow and the size of the bubbles.

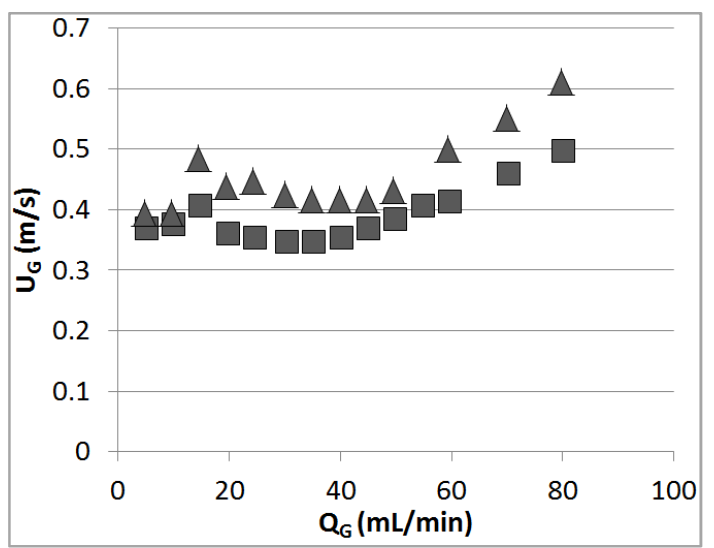

Figure 8. Bubble velocity versus $\mathrm{CO}_{2}$ flow.

\subsection{PIV Measures}

In order to characterize the flow and the turbulence in the aquarium vertical velocity vectors are obtained using Velocimetry Particle Image (PIV) or Particle Image Velocimetry.

Frequency, exposure and recording range

The recording frequency of the DALSA camera is a function of the vertical recording height figure 9. However, the recording width does not influence the frequency. There must be a compromise between the recording range and the recording frequency. On the other hand if the recording frequency increases the maximum exposure time decreases. Exposure time greater than $2500 \mu$ s results in blurred bubble outlines. On the other hand a significant exposure time allows a better brightness.

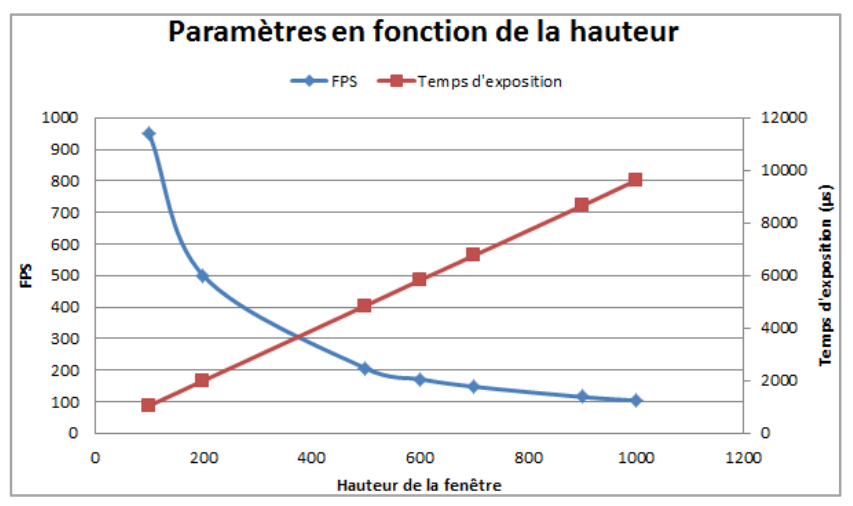

Figure 9. Recording Settings by Height.

These images are then processed to calculate the average 
intensity, using a matlab program, giving the average gray level on a group of images. The results give the figure 10 . On this curve, the peak of intensity appears very clearly for a concentration of $7.00 \mathrm{E}-06 \mathrm{~mol} \cdot \mathrm{L}^{-1}$, the intensity having gradually increased with the concentration until then. Past this peak, the intensity decreases quite linearly. This decay corresponds to fluorescein particles which hide themselves, blocking the light rays for high concentrations. Note a hook for a concentration of $1.30 \mathrm{E}-06 \mathrm{~mol} \cdot \mathrm{L}-1$ fluorescein, which corresponds to a slight change on the diaphragm of the camera during handling.

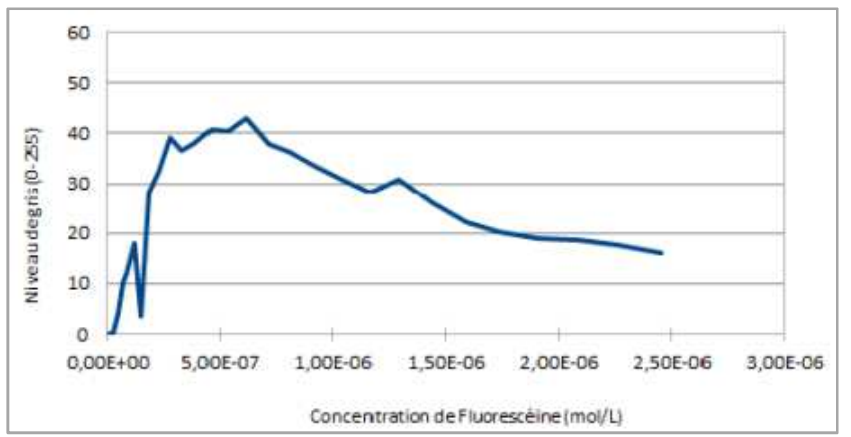

Figure 10. Intensity of fluorescein as a function of its concentration.

\section{Fluorescein}

Fluorescein is a dye which has the particularity of returning a fluorescent light when excited at a wavelength of $494 \mathrm{~nm}$. However, this fluorescence depends on the dye concentration. One might think that the more concentrated it is, the more fluorescent it is. However, a study carried out under this project shows that after a stage, the fluorescence tends to disappear. The manipulation consists in gradually increasing the concentration of fluorescein in the tank, illuminated by a laser plane and recording the images. The images obtained have intensity varying with concentration (Figure 11).

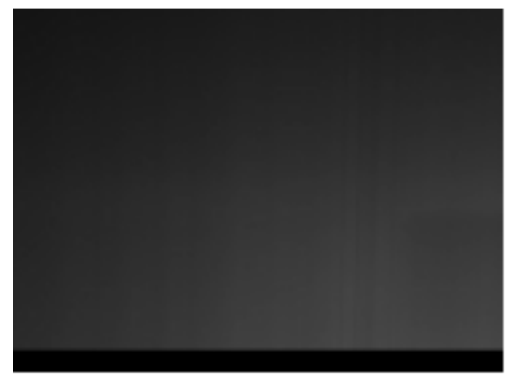

a) with $120 \mathrm{~mL}$ of fluorescein

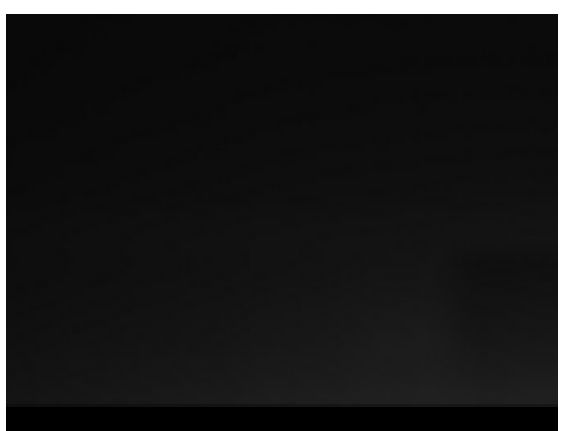

b) with $490 \mathrm{~mL}$ of fluorescein

Figure 11. Mean image with different amount of fluorescein.

\section{Principle of the PIV}

Particle Image Velocimetry is an optical method of determining the velocity of a fluid from the movement of particles carried by it. This technique allows to reconstruct a velocity field in a plane by dividing the images into interrogation windows and by determining the velocity of the particles located therein (figure 12). At present, optical techniques are preferred for turbulence measurements because the sensor is located outside the flow. They are said to be non-intrusive and do not disturb the flow [11].

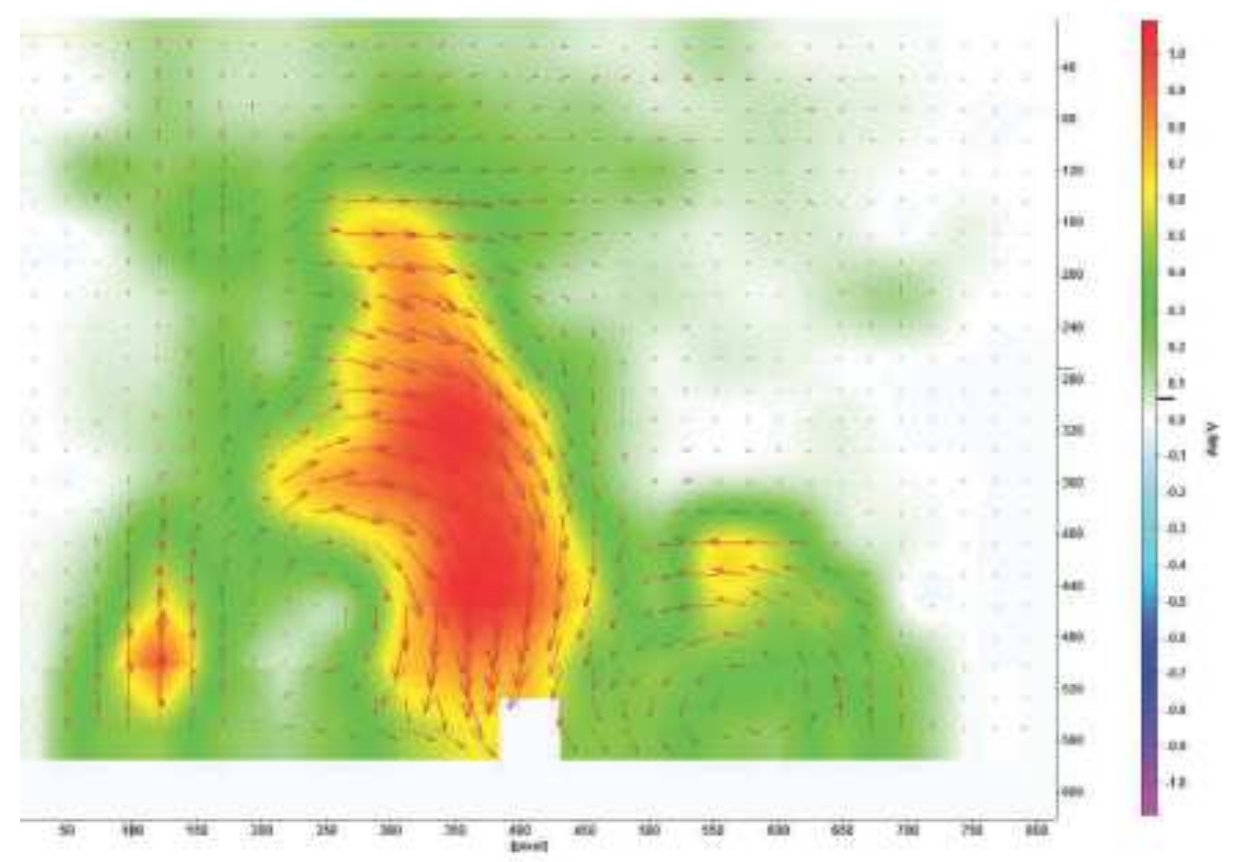

Figure 12. Velocity field measured by PIV. 


\section{Conclusion}

The aquarium is a device that ensures a mixture between a bubble-shaped gas phase and a liquid phase. The use of PIV experimental techniques has allowed for a better understanding of the hydrodynamics of two-phase flow. For some decades, the studies carried out on this device have contributed to the understanding of their hydrodynamics [12]. The gas-liquid flows in these columns are intrinsically unstable and the dynamics of such flows influence mixing and mass transfer performance. The hydrodynamics of the liquid phase illustrates that the liquid flow rate in the aquarium is directly proportional to the injected gas flow rate. It is therefore important to characterize the dynamics of the gas-liquid flow [13]. Also, the complete knowledge of the global dynamics of the aquarium fluids is based on that of the bubble [14]. Knowledge of bubble size and vacuum ratio is crucial for determining gas-liquid interfacial air and therefore mass transfer [15]. The non-intrusive techniques used is the image analysis by PIV $[11,16]$. The influence of the extinction coefficient changes was observed on $\mathrm{CO}_{2}$ concentration during tests in the wake of a free-climbing bubble in a water column.

\section{References}

[1] J. Chaouki, et al., noninvasive tomographic and velocimetric monitoring of multiphase flows. Ind. Eng. Chem. Res. 36, (1997) 4476-503.

[2] A. H. Barkaï et al., Freshwater Purification by Vacuum Airlift Column Using Methyl Isobutyl Carbinol and Casein. Open Journal of Applied Sciences, 2019, 9, 230-239.

[3] D. Bongo et al., Study of the Transfer of $\mathrm{CO}_{2}-\mathrm{H}_{2} \mathrm{O}$ Mass in an Aquarium under the Influence of one Oscillating Railing. IJIRSET. Vol. 6, Issue 10, October 2017.

[4] B. Barru, et al., Mass transfer efficiency of a vacuum airlift Application to water recycling in aquaculture systems. Aquacult. Eng (2011).
[5] B. Barru. Etude et optimisation du fonctionnement d'une colonne airlift à dépression - Application à l'aquaculture. (2011).

[6] A. H. Barkaï et al., Etude par bi-sonde optique d'un écoulement à bulles d'une colonne air-lift sous dépression. 23 ème Congrès Français de Mécanique, 2017.

[7] Albani, J. R. (2001). Absorption et fluorescence: principes et applications. Paris, tec \& doc edition.

[8] Yu W., Wang T. F., Liu M., Wang Z. W., 2008. Bubble Circulation Regimes in a Multi-Stage Internal-Loop Airlift Reactor. Chem. Eng. J. 142, 301-30.

[9] Chaumat, H., Billet-Duquenne, Delmas, H., 2007b. Hydrodynamics and mass transfer in bubble column: Influence of liquid phase surface tension. Chemical Engineering Science 62, 7378-7390.

[10] Painmanakul, P., Loubière, K., Hébrard, G., Mietton-Peuchot, M., Roustan, M., 2005. Effect of surfactants on liquid-side mass transfer coefficients, Chemical Engineering Science 60, 6480-6491.

[11] H. Chaumat, et al., on the reliability of an optical fibre probe in bubble column under industrial relevant operating conditions. Exp. Therm. Fluid Sci. 31, 2007, 495-504.

[12] B. K. Singh, et al., Dynamics of gas-liquid flow in a cylindrical bubble column: Comparison of electrical resistance tomography and voidage probe measurements. Chem. Eng. Sci. 158 (2017) 124-139.

[13] P. Zehner, and M. Kraume, 2000. Bubble Columns. Ullmann's Encyclopedia of Industrial Chemistry.

[14] G. Besagni, et al., The dual effect of viscosity on bubble column hydrodynamics. Chemical Engineering Science 158 (2017) 509-538.

[15] B. J. Azzopardi, al., Bubble columns, in: Hydrodynamics of Gas-Liquid Reactors: Normal Operation and Upset Conditions, John Wiley \& Sons, Ltd, 2011.

[16] S. Besbes, al., PIV measurements and Eulerian-Lagrangian simulations of the unsteady gas-liquid flow in a needle sparger rectangular bubble column. Chem. Eng. Sci. 126, (2015) 560-572. 